\title{
Effet de la canicule de 2003 sur les poissons migrateurs en Garonne et Dordogne - Réflexions sur l'effet des rejets thermiques de la centrale nucléaire de Golfech sur la Garonne
}

\author{
Effect of the 2003 heat wave on the migratory fish \\ in the Garonne and Dordogne rivers - Consideration \\ on the effect of the Golfech nuclear power plant \\ thermal discharges in the Garonne river
}

\author{
F. Travade(1), L. Carry(2) \\ (1) EDF R\&D, Département LNHE, 6 quai Watier, 78400 Chatou, France \\ (2) MIGADO, 35 avenue de la Marqueille, 31650 Saint-Orens, France \\ e-mail : francois.travade@edf.fr
}

Résumé - L'effet de la canicule de l'été 2003 en France sur quatre espèces de poissons migrateurs diadromes (le saumon Atlantique - Salmo salar, l'alose - Alosa alosa, l'anguille Anguilla anguilla et la lamproie marine - Petromyzon marinus) de la Garonne et de la Dordogne est analysé à partir des données de passage des migrateurs dans deux stations de comptage et à partir de diverses observations réalisées lors de cette épisode caniculaire. La station de comptage de la Garonne (Golfech) est située à proximité immédiate des rejets d'une centrale nucléaire alors que celle de la Dordogne est indemne de rejets thermiques. L'épisode caniculaire s'est traduit par des modifications des rythmes saisonniers de migrations, des modifications des migrations annuelles et par des mortalités variables selon les espèces considérées. La similarité des phénomènes sur la Garonne et la Dordogne laisse à penser que l'effet des rejets thermiques de la centrale nucléaire a été faible, voire inexistant.

Mots clés - canicule, poissons migrateurs, rejet thermique, rivière, Garonne, Dordogne

Abstract - The effect of the summer 2003 heat wave in France on four species of migratory diadromous fish (Atlantic salmon - Salmo salar, shad - Alosa alosa, eel - Anguilla anguilla and sea lamprey - Petromyzon marinus) of the river Garonne and the river Dordogne is analyzed from data of migratory fish passage in two fish counting stations and various observations made during this heat wave period. The counting station of the Garonne (Golfech) is located near the discharge of a nuclear power plant while that of the Dordogne is exempt of thermal discharges. The heat wave event has resulted in changes in seasonal migration patterns, changes in annual migration and in mortality depending on fish species. The similarity of phenomena on the Garonne and the Dordogne suggests that the effect of the nuclear power station thermal discharge is low or nonexistent

Key words - heat wave, migratory diadromous fish, thermal discharge, river, Garonne, Dordogne 


\section{INTRODUCTION - PROBLÉMATIQUE}

La canicule de 2003, en France, s'est traduite par des températures printanières et estivales très élevées ayant conduit à des températures de l'eau voisines ou supérieures à $30^{\circ} \mathrm{C}$ sur la plupart des grands cours d'eau. Ces températures élevées se sont accompagnées en général de faibles débits fluviaux.

L'effet de ces températures extrêmes sur les migrateurs diadromes qui fréquentent nos cours d'eau pose question du fait que les migrations de remontée dans les cours d'eau se situent pour la plupart d'entre eux au printemps et en été. Par ailleurs, concernant la Garonne, s'est posée également la question de l'incidence supplémentaire des rejets thermiques de la centrale nucléaire de Golfech.

Pour répondre à ces deux questions nous avons analysé les données de migration et recueilli les observations disponibles sur la Garonne et la Dordogne pour quatre espèces de migrateurs diadromes, le saumon Atlantique (Salmo salar), la grande alose (Alosa alosa), l'anguille (Anguilla anguilla) et la lamproie marine (Petromyzon marinus).

\section{MÉTHODOLOGIE}

L'analyse repose pour partie sur les passages de migrateurs dans les stations de contrôle des passes à poissons des centrales hydroélectriques EDF de Golfech sur la Garonne et de Tuilières sur la Dordogne et pour partie sur les diverses observations et analyses effectuées sur les migrateurs des deux axes fluviaux durant la période caniculaire.
La passe à poissons de Golfech est un ascenseur assurant le franchissement de l'usine hydroélectrique située à quelques centaines de mètres en amont des rejets de la centrale nucléaire de Golfech. Celle de Tuilières est également un ascenseur situé à une vingtaine de kilomètres en amont de Bergerac. Le site n'est pas influencé par un échauffement de centrale thermique. La distance des deux sites par rapport à l'estuaire de la Gironde (bec d'Ambès) est respectivement de $200 \mathrm{~km}$ pour Golfech et de $100 \mathrm{~km}$ pour Bergerac (Fig. 1), ce qui peut modifier quelque peu les dates de début de passage des migrateurs dans les dispositifs.

Les comptages de migrateurs, effectués par l'association MIGADO (MIGADOa 1992 à 2007; MIGADOb 1992 à 2007), sont réalisés à l'aide de dispositifs vidéo, qui consistent à filmer les passages de poissons derrière des vitres installés dans les deux passes (Photos A et B). Ces comptages sont effectués en permanence (24 heures sur 24) depuis 1987 à Golfech et depuis 1989 à Tuilières. Pour l'anguille, les comptages postérieurs à 1997 pour Tuilières et 2002 pour Golfech ont été effectués par un compteur automatique à résistivité installé sur les passes spécifiques à anguille lors de leur construction (Photo C). L'ensemble des comptages permet de disposer de séries chronologiques des rythmes de migration de l'ensemble des poissons à un pas de temps horaire.

Pour les quatre espèces migratrices considérées (saumon, alose, anguille et lamproie), nous avons comparé, sur chacun des sites, les passages de 2003 à ceux de la série chronologique à l'échelle annuelle (chronique 


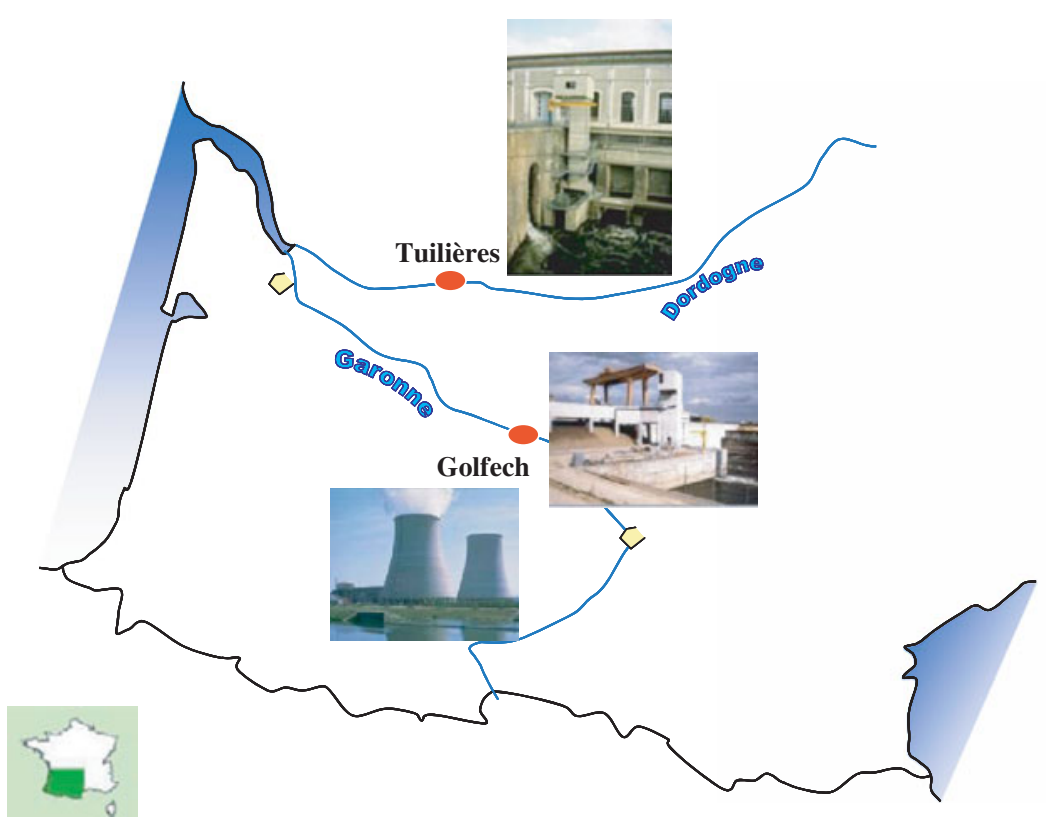

Fig. 1. Localisation des stations de contrôle des migrateurs de Tuilières et Golfech et de la centrale nucléaire de Golfech.

Fig. 1. Location of the migratory fish counting stations of Tuilieres and Golfech and location of the Golfech nuclear power station.

1987-2007 pour Golfech et 1989-2005 pour Tuilières) et à l'échelle hebdomadaire de façon à examiner l'effet des températures élevées sur le déroulement de la migration au cours de l'année 2003.

Les débits fluviaux proviennent de la station de jaugeage DDE de La Magistère pour la Garonne, et de la centrale de Tuilières pour la Dordogne.

Les températures de l'eau proviennent pour partie de la station de mesure multi-paramètres de la centrale nucléaire de Golfech (station amont), et pour partie des thermographes (MIGADO et EDF R\&D) implantés dans les passes à poissons de Golfech et Tuilières.

\section{RÉSULTATS}

\subsection{Débits et températures lors de la canicule}

Les débits sur les deux axes fluviaux sont très faibles à compter du printemps 2003 (Figs. 2a et 2b). Sur la Garonne à Golfech le débit est inférieur aux minima de la série de référence (1992-2002) dans la période du 3 juillet au 8 septembre avec un minimum de $40 \mathrm{~m}^{3} / \mathrm{s}$ les 7 et 8 août. Sur la Dordogne à Tuilières l'étiage sévère est de plus longue durée que sur la Garonne; le débit est inférieur aux minima de la série de référence 


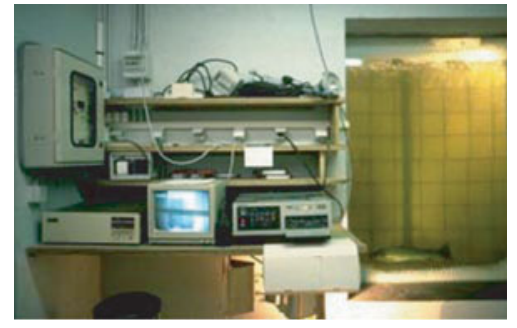

A

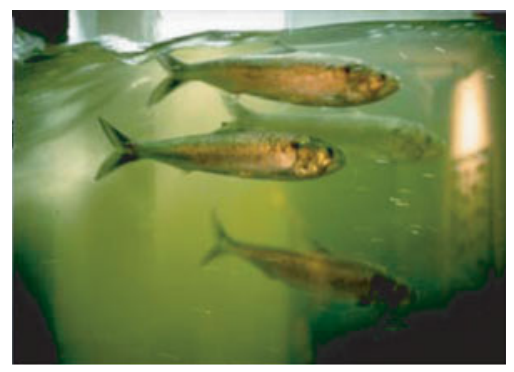

B

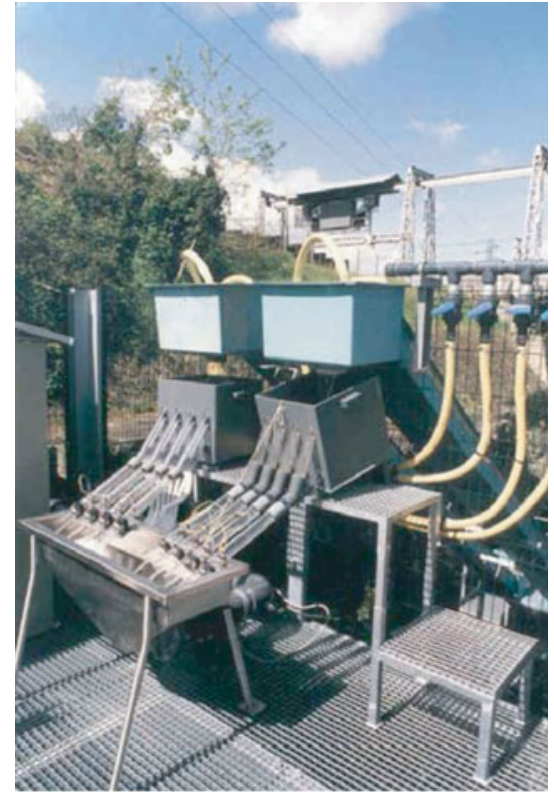

$\mathrm{C}$

Photo 1. Station de comptage vidéo ( $A$ et $B$ ) et compteur automatique à anguille $(C)$.

Photo 1. Video fish counting station (A and $B$ ) and automatic eel counting device (C).

(1993-2002) dans la période du 11 mai au 16 octobre avec un minimum de $15 \mathrm{~m}^{3} / \mathrm{s}$ du 9 au 17 août.

Les régimes thermiques (Figs. 3a et $3 b$ ), similaires sur les deux sites sont caractérisés par une augmentation rapide des températures de l'eau dès le mois de mai : température de $16^{\circ} \mathrm{C}$ atteinte fin-avril - début-mai, soit 2 à 3 semaines plus tôt par rapport à la série de référence. Les températures augmentent plus fortement à compter de début-juin pour atteindre $25^{\circ} \mathrm{C}$ le 9 juin sur la Dordogne et le 27 juin sur la Garonne puis atteindre ou dépasser $30^{\circ} \mathrm{C}$ à compter du 9 août à Tuilières et du 12 août à Golfech. Les maxima observés sont similaires sur les deux cours d'eau : $30,8^{\circ} \mathrm{C}$ (moyenne journalière) et $32^{\circ} \mathrm{C}$ (valeur instantanée) à Golfech; $30^{\circ} \mathrm{C}$ (moyenne journalière) et $32^{\circ} \mathrm{C}$ (valeur instantanée) à Tuilières. La température reste supérieure à $25^{\circ} \mathrm{C}$ sur les deux cours d'eau jusqu'au 30 août.

À l'aval de la centrale nucléaire de Golfech, les mesures et modèles thermiques réalisés lors de l'épisode de canicule font apparaître deux phénomènes (Hendrickx et al., 2003) :

- un échauffement dû à la centrale de l'ordre de $0,7^{\circ} \mathrm{C}$. La température maximale instantanée enregistrée à l'aval immédiat de la centrale a été de $31,2^{\circ} \mathrm{C}$ le 12 août, 
GARONNE à GOLFECH

Débits en 2003 et sur la période 1992-2002

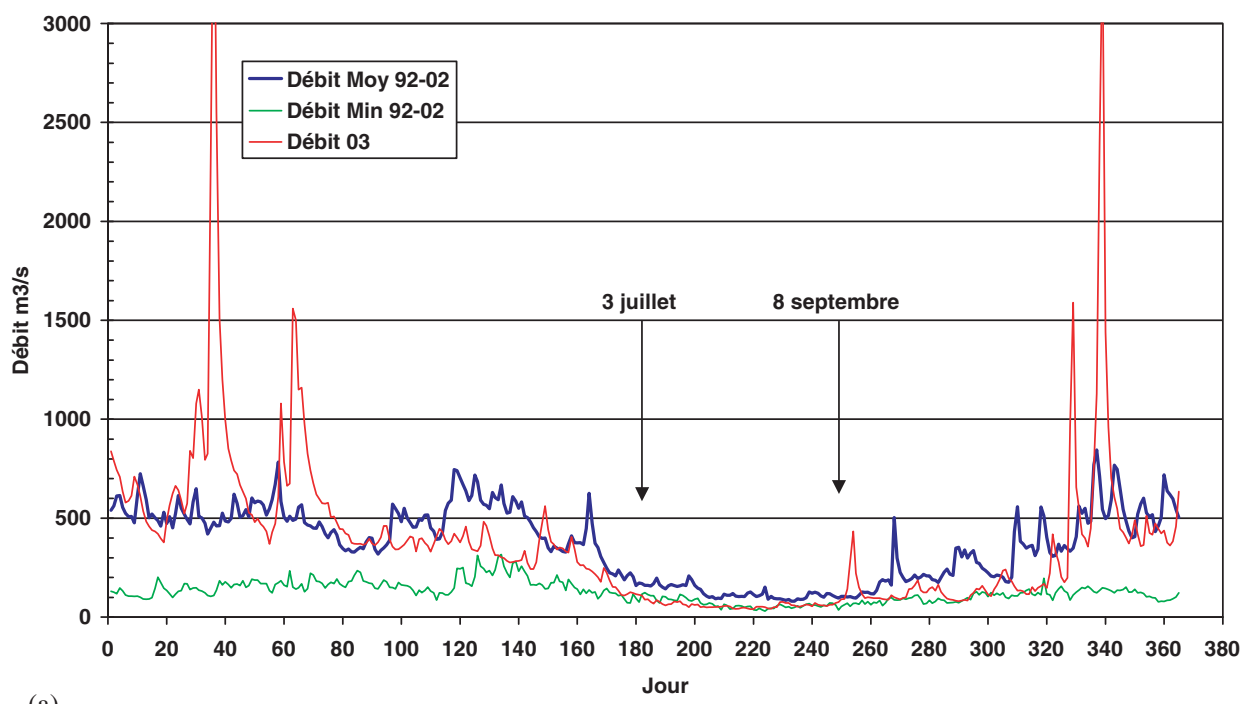

(a)

DORDOGNE à TUILIERES

Débits en 2003 et sur la période 1993-2002

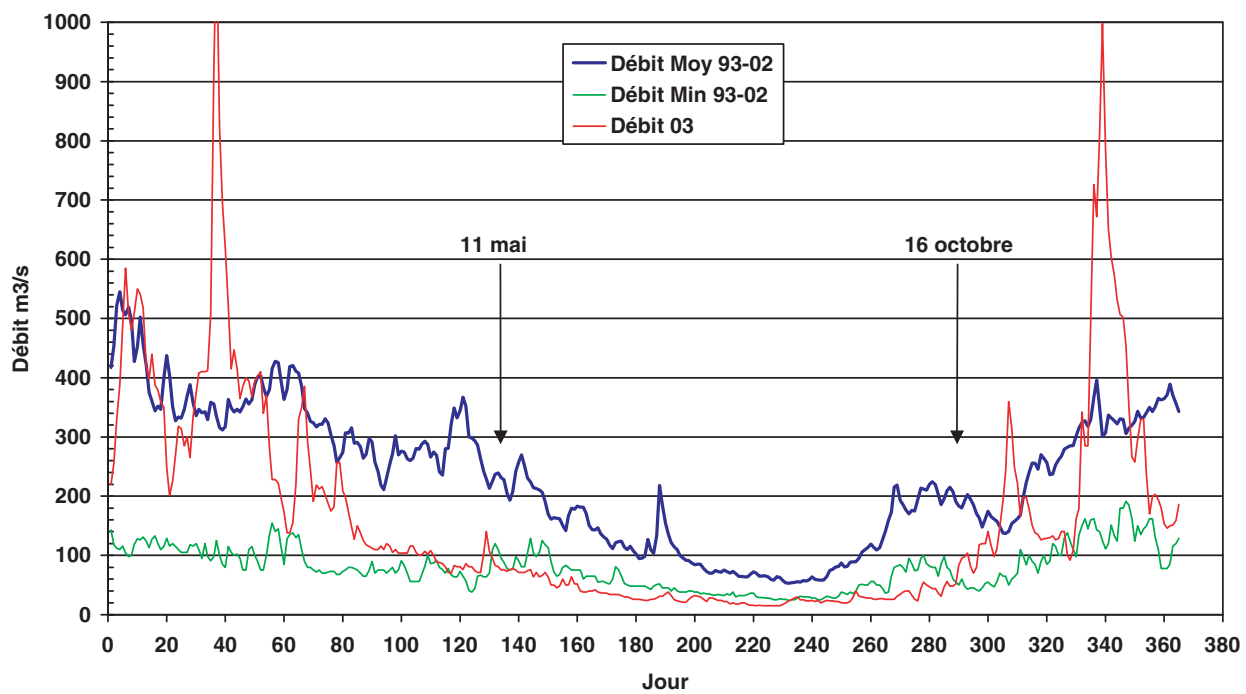

(b)

Fig. 2. (a) Débit de la Garonne à Golfech en 2003 et sur la période de référence 1992-2002; (b) débit de la Dordogne à Tuilières en 2003 et sur la période de référence 1993-2002.

Fig. 2. (a) Discharge of the river Garonne at Golfech in 2003 and during the reference period 19922002; (b) discharge of the river Dordogne at Tuilieres in 2003 and during the reference period 19932002. 
GARONNE à GOLFECH

Température de l'eau en 2003 et sur la période 1992-2002

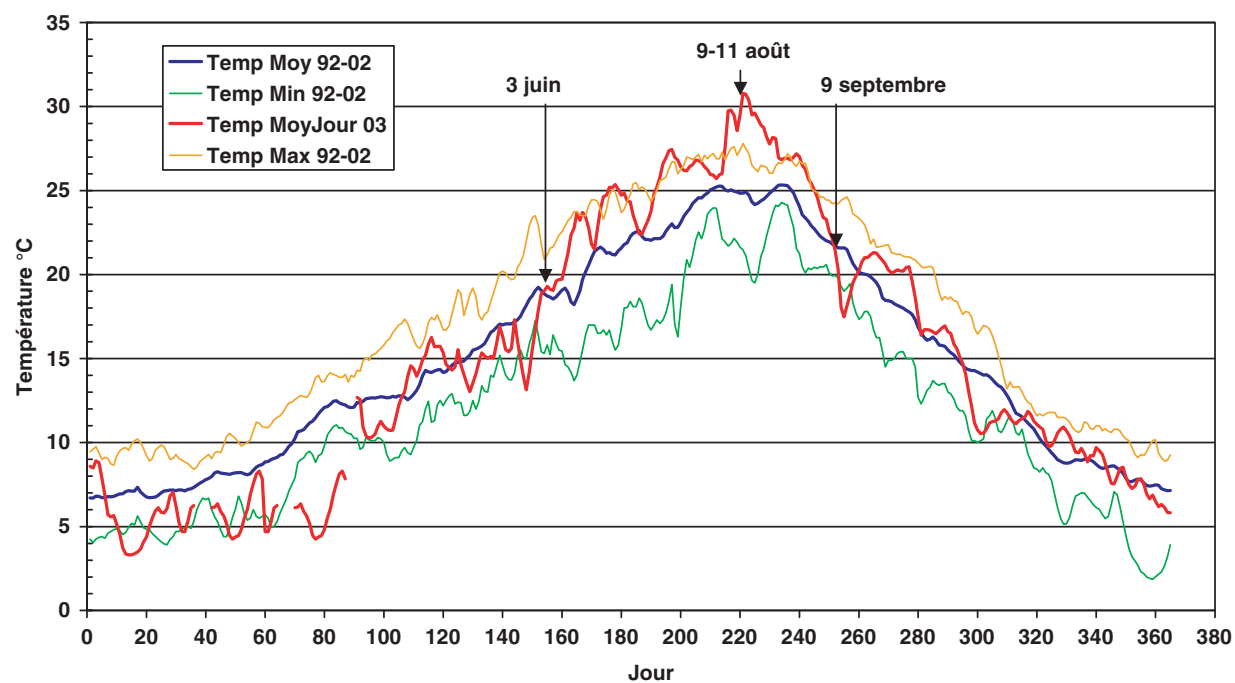

(a)

DORDOGNE à TUILIERES

Température de l'eau en 2003 et sur la période 1993-2002

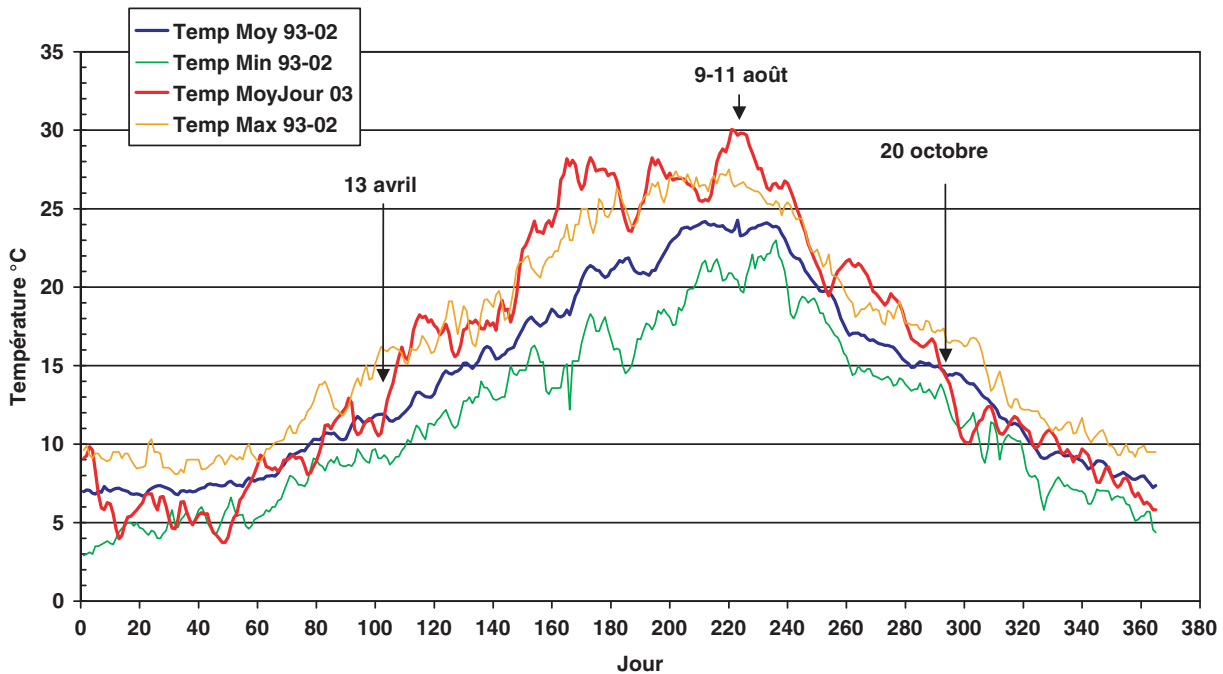

(b)

Fig. 3. (a) Température de la Garonne à Golfech en 2003 et sur la période de référence 1992-2002; (b) température de la Dordogne à Tuilières en 2003 et sur la période de référence 1993-2002.

Fig. 3. (a) Temperature of the river Garonne at Golfech in 2003 and during the reference period 1992-2002; (b) temperature of the river Dordogne at Tuilieres in 2003 and during the reference period 1993-2002. 
- un échauffement naturel dû à la mise à l'équilibre de la masse d'eau (qui ne l'est pas à l'amont de Golfech du fait de la présence de la retenue de Malause) avec les paramètres atmosphériques locaux qui se produit sur plusieurs dizaines de kilomètres à l'aval de la centrale. Une température maximale de $32,5^{\circ} \mathrm{C}$ a été mesurée le 12 août à $15 \mathrm{~km}$ à l'aval de la centrale. La température maximale à $40 \mathrm{~km}$ à l'aval de la centrale, calculée par le modèle thermique, aurait été de $33,3^{\circ} \mathrm{C}$.

\subsection{Saumon Atlantique}

À l'échelle annuelle (Tabs. I et II, Figs. $4 a$ et $4 b$ ), les passages de saumons sur la période de référence (1989-2002) sont similaires pour la Garonne et la Dordogne et se caractérisent par une augmentation graduelle des migrations, vraisemblablement représentative de la restauration de la population de saumon sur les deux axes. Le maximum atteint est de 599 individus à Golfech en 2001 et de 1417 individus à Tuilières en 2002 . En 2003, on observe une diminution très

Tableau I. Dénombrement des migrateurs dans la station de comptage de l'ascenseur de Golfech sur la Garonne (données MIGADO).

Table I. Passages of migratory fish at the counting station of the Golfech fish lift on the river Garonne.

\begin{tabular}{lllll}
\hline Année & $\begin{array}{l}\text { Alose } \\
\text { Alosa alosa }\end{array}$ & $\begin{array}{l}\text { Anguille } \\
\text { Anguilla anguilla (a) }\end{array}$ & $\begin{array}{l}\text { Lamproie marine } \\
\text { Petromyzon marinus }\end{array}$ & $\begin{array}{l}\text { Saumon atlantique } \\
\text { Salmo salar (a) }\end{array}$ \\
\hline $\mathbf{1 9 8 7}$ & 18224 & $4970(\mathrm{a})$ & 11 & 24 \\
$\mathbf{1 9 8 8}$ & 13779 & $1166(\mathrm{a})$ & 16 & 25 \\
$\mathbf{1 9 8 9}$ & 66401 & $13322(\mathrm{a})$ & 370 & 1 \\
$\mathbf{1 9 9 0}$ & 43000 & $?$ & $?$ & 4 \\
$\mathbf{1 9 9 1}$ & 40074 & $27819(\mathrm{a})$ & 200 & 7 \\
$\mathbf{1 9 9 2}$ & 20007 & $172(\mathrm{a})$ & 747 & 14 \\
$\mathbf{1 9 9 3}$ & 18554 & $288(\mathrm{a})$ & 2086 & 47 \\
$\mathbf{1 9 9 4}$ & 85813 & $4482(\mathrm{a})$ & 107 & 134 \\
$\mathbf{1 9 9 5}$ & 85624 & $1460(\mathrm{a})$ & 741 & 116 \\
$\mathbf{1 9 9 6}$ & 106705 & $2009(\mathrm{a})$ & 2382 & 61 \\
$\mathbf{1 9 9 7}$ & 98819 & $3986(\mathrm{a})$ & 663 & 93 \\
$\mathbf{1 9 9 8}$ & 49074 & $164(\mathrm{a})$ & 1618 & 258 \\
$\mathbf{1 9 9 9}$ & 36373 & $59(\mathrm{a})$ & 222 & 433 \\
$\mathbf{2 0 0 0}$ & 32584 & $49(\mathrm{a})$ & 789 & 599 \\
$\mathbf{2 0 0 1}$ & 25277 & $18(\mathrm{a})$ & 219 & 354 \\
$\mathbf{2 0 0 2}$ & 17460 & $31236(\mathrm{~b})$ & 4147 & $\mathbf{8 2}$ \\
$\mathbf{2 0 0 3}$ & $\mathbf{2 2} 269$ & $\mathbf{1 0 1 9 2 6 ( b )}$ & $\mathbf{1 8 3 4 4}$ & 126 \\
$\mathbf{2 0 0 4}$ & 19993 & $27505(\mathrm{~b})$ & 2834 & 47 \\
$\mathbf{2 0 0 5}$ & 18306 & $68200(\mathrm{~b})$ & 2132 & 128 \\
$\mathbf{2 0 0 6}$ & 9671 & $36000(\mathrm{~b})$ & 434 & 148 \\
$\mathbf{2 0 0 7}$ & 2979 & $103592(\mathrm{~b})$ & 5626 & \\
\hline
\end{tabular}

(a) Comptage non fiable de 1987 à 2001 = indication d'abondance; (b) passages cumulés dans l'ascenseur et la passe spécifique à anguille installée en 2002. 
Tableau II. Dénombrement des migrateurs dans la station de comptage de l'ascenseur de Tuilières sur la Dordogne (données MIGADO).

Table II. Passages of migratory fish at the counting station of the Tuilieres fish lift on the river Dordogne.

\begin{tabular}{lllll}
\hline Année & $\begin{array}{l}\text { Alose } \\
\text { Alosa alosa }\end{array}$ & $\begin{array}{l}\text { Anguille } \\
\text { Anguilla anguilla }\end{array}$ & $\begin{array}{l}\text { Lamproie marine } \\
\text { Petromyzon marinus }\end{array}$ & $\begin{array}{l}\text { Saumon atlantique } \\
\text { Salmo salar }\end{array}$ \\
\hline $\mathbf{1 9 8 9}$ & 7789 & $18887(\mathrm{a})$ & 1111 & 3 \\
$\mathbf{1 9 9 0}$ & 4943 & $4014(\mathrm{a})$ & 36 & 19 \\
$\mathbf{1 9 9 1}$ & 6053 & $13496(\mathrm{a})$ & 1350 & 9 \\
$\mathbf{1 9 9 2}$ & 1945 & $16301(\mathrm{a})$ & 909 & 82 \\
$\mathbf{1 9 9 3}$ & 35704 & $14592(\mathrm{a})$ & 6693 & 85 \\
$\mathbf{1 9 9 4}$ & 62592 & $13344(\mathrm{a})$ & 4368 & 334 \\
$\mathbf{1 9 9 5}$ & 78245 & $3207(\mathrm{a})$ & 4419 & 96 \\
$\mathbf{1 9 9 6}$ & 87254 & $5075(\mathrm{a})$ & 2923 & 296 \\
$\mathbf{1 9 9 7}$ & 42374 & $15438(\mathrm{~b})$ & 2913 & 526 \\
$\mathbf{1 9 9 8}$ & 28465 & $18246(\mathrm{~b})$ & 4223 & 195 \\
$\mathbf{1 9 9 9}$ & 63308 & $13108(\mathrm{~b})$ & 4367 & 1055 \\
$\mathbf{2 0 0 0}$ & 48751 & $10376(\mathrm{~b})$ & 9820 & 1023 \\
$\mathbf{2 0 0 1}$ & 50828 & $45118(\mathrm{~b})$ & 5093 & 1417 \\
$\mathbf{2 0 0 2}$ & 39528 & $33039(\mathrm{~b})$ & 11435 & $\mathbf{1 8 4}$ \\
$\mathbf{2 0 0 3}$ & $\mathbf{2 3} \mathbf{8 3 5}$ & $\mathbf{3 3} 109$ (b) & $\mathbf{3 0 2 6 5}$ & 122 \\
$\mathbf{2 0 0 4}$ & 30106 & $23146(\mathrm{~b})$ & 38762 & 306 \\
$\mathbf{2 0 0 5}$ & 15975 & $22454(\mathrm{~b})$ & 21052 & \\
\hline
\end{tabular}

(a) Passages dans l'ascenseur à poisons seul (efficacité limitée pour l'anguille); (b) passages dans l'ascenseur à poissons et la passe spécifique anguille construite en 1997.

importante des passages sur les deux sites : 88 et 177 individus respectivement à Golfech et Tuilières, soit une réduction des passages du facteur 4 (Golfech) à 8 (Tuilières) par rapport aux passages de l'année 2002. Dans la période qui suit la canicule, les passages restent à un niveau bas sur les deux sites et ne reviennent pas aux niveaux antérieurs à 2002.

À l'échelle hebdomadaire, on observe, sur la série de référence (1992-2002), que la migration du saumon sur les deux cours d'eau est caractérisée par trois périodes de migration, classiques pour cette espèce (Baglinière et Porcher, 1994) (Figs. 5 et 6) :

- migration d'hiver et printemps de janvier à mi-juin (semaines 1 à
24) constituée de grands individus qui ont passé deux à trois hivers en mer,

- migration d'été de mi-juin à août (semaines 24 à 33), constituée de petits individus (castillons ou madeleineaux), ayant passé un seul hiver en mer,

- migration d'automne, comprenant des saumons de un ou deux hivers de mer.

À Golfech (Fig. 5), il existe un arrêt estival d'environ 2 mois entre la période de migration estivale et automnale alors qu'à Tuilières (Fig. 6) cet arrêt de migration est plus bref (2 semaines environ) voire inexistant certaines années. Cette différence entre les deux sites est vraisemblablement due aux 

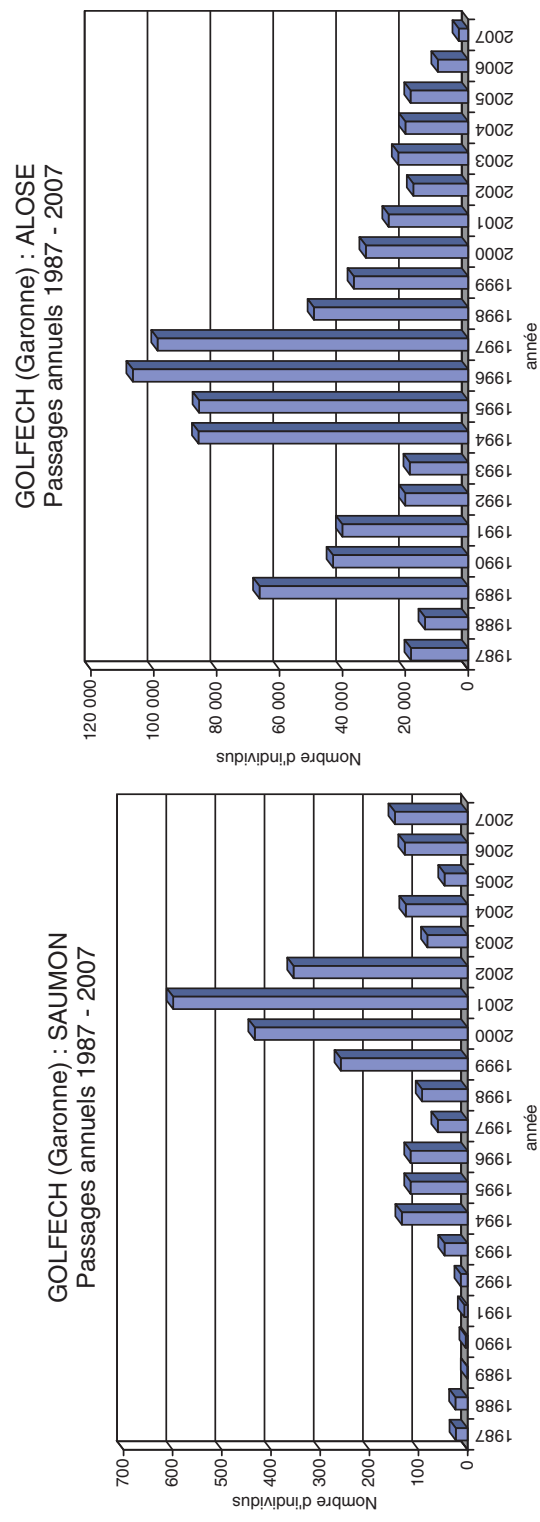
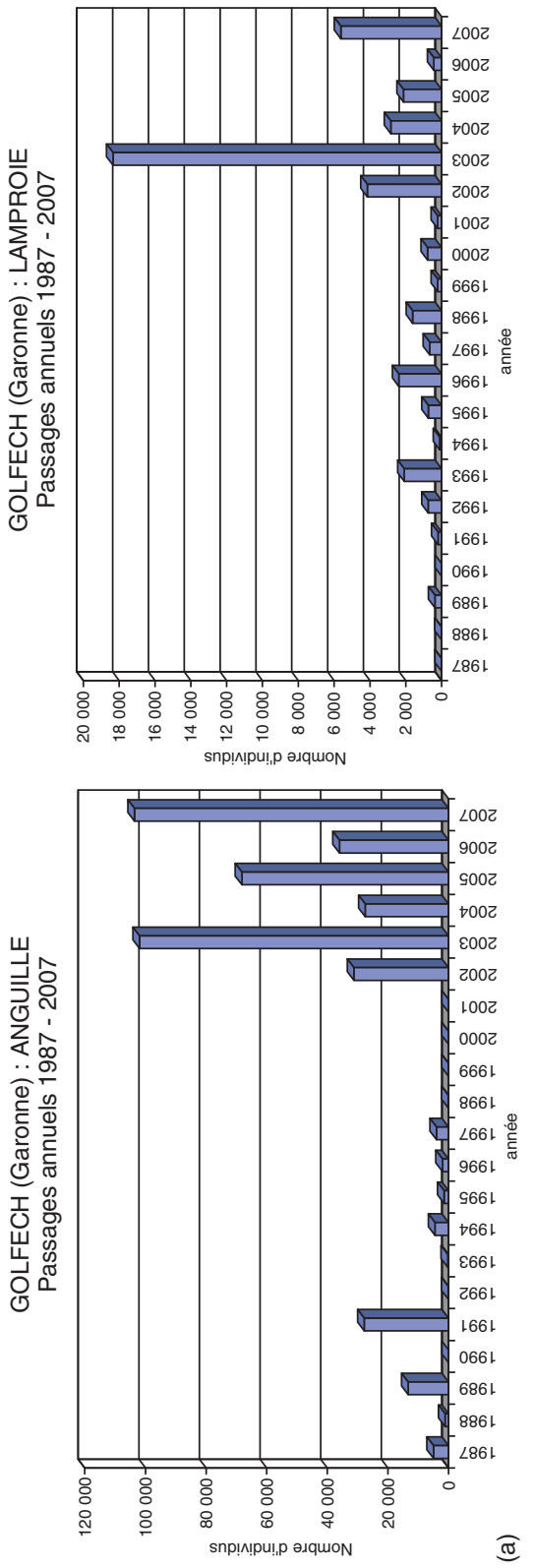

\& $\frac{\Gamma}{6}$

代

$\stackrel{2}{\frac{\pi}{\pi}}$

a

穴

$\pi$ ब

อ

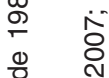

눈

히 क

ธ

$\div$

क

웡 윰

(1)

잉

ำ

ผ เ

응ㅇㅇ 용

-

亏 के

(1) 은 은

웡

ธ 0 ठ

항 등

๓ 은 똥

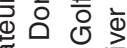

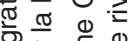

के

(2) 0 is

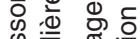

高

का क

क ⿻ 丨

के क्ञ

$\frac{\omega}{\Phi} \stackrel{0}{\mathbb{2}} \geq$

흥

ट

ஸ) क्.

定

政 $\frac{\sigma}{2}$

कृ 을

둥

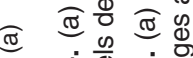

+ $\frac{0}{4}+\dot{8}$

ㅎํㄴ 들 원 ه 

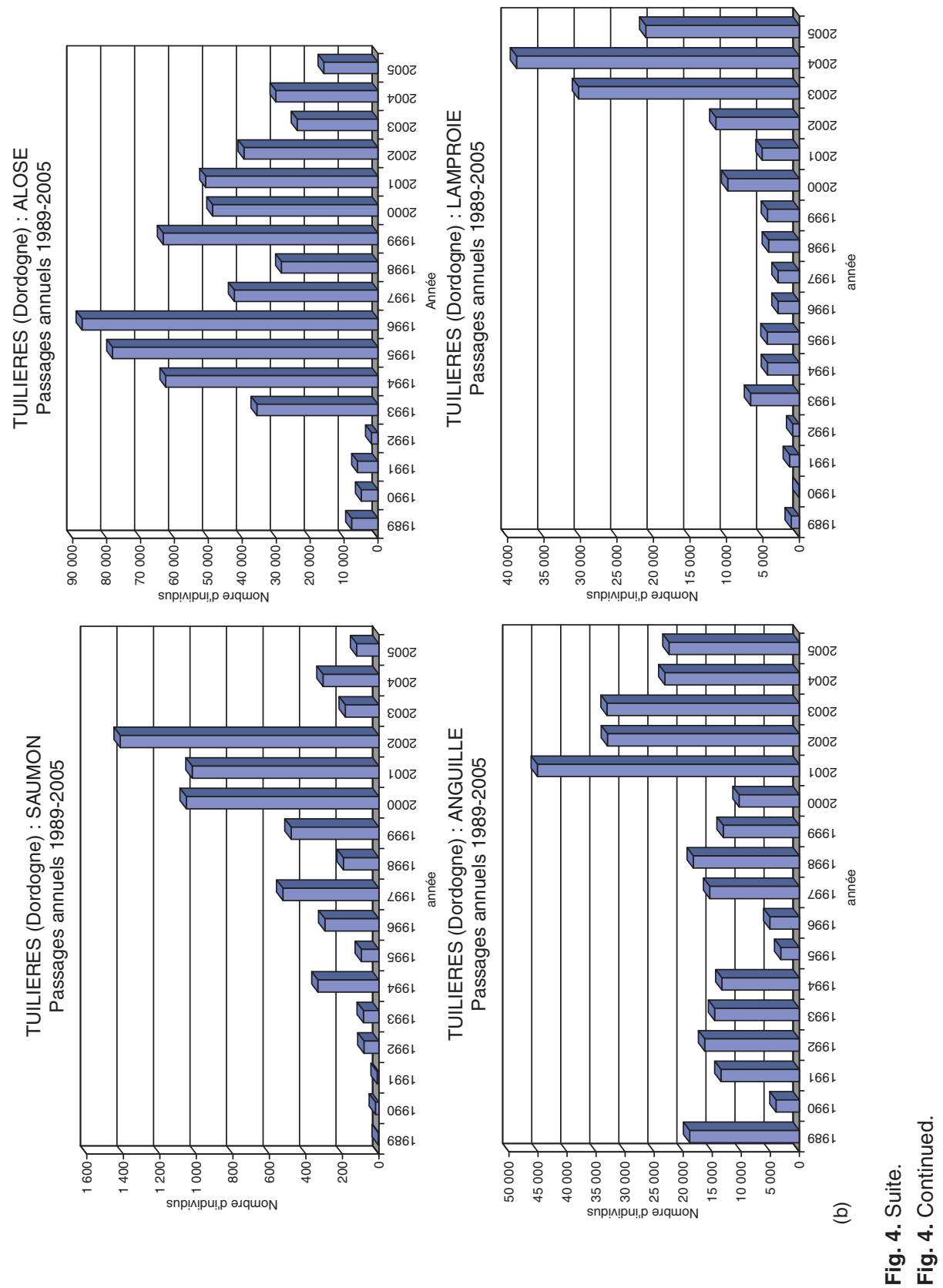


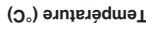
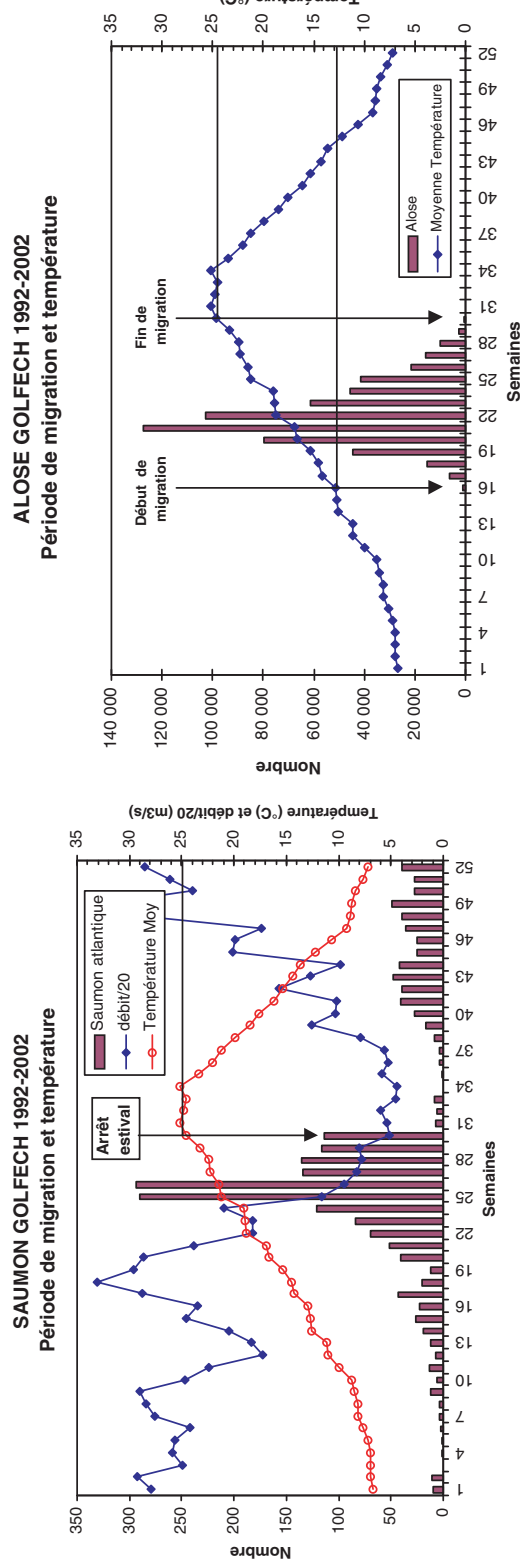

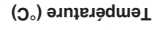
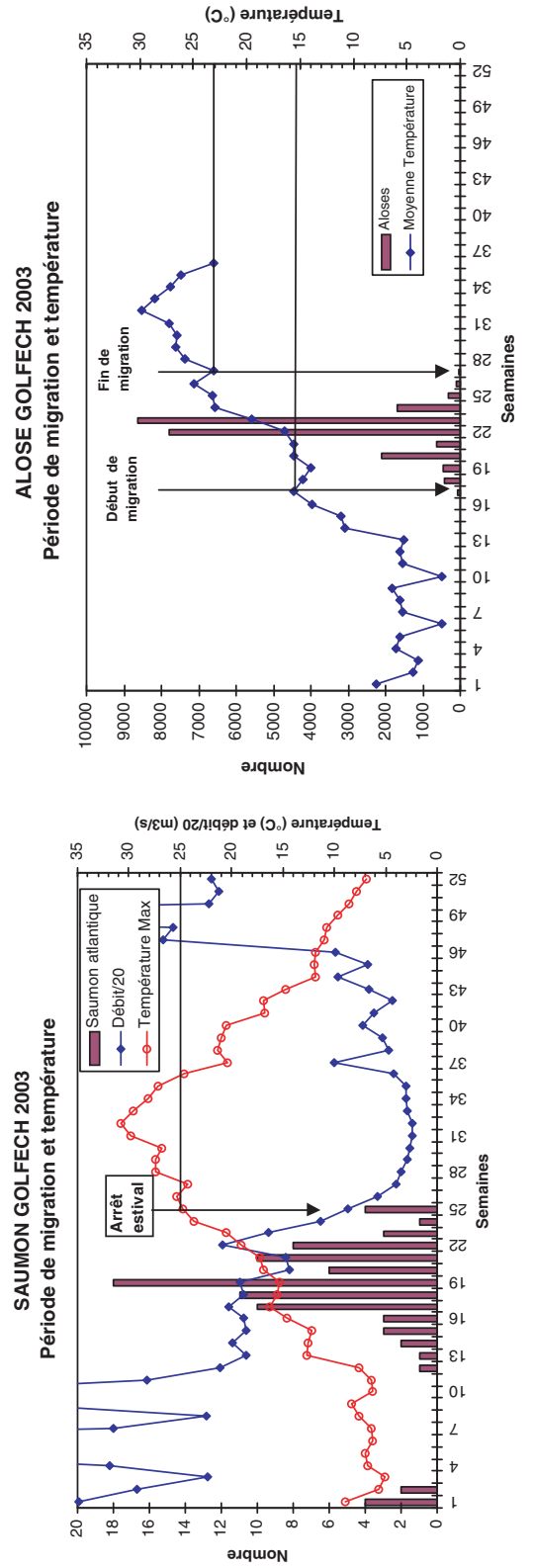

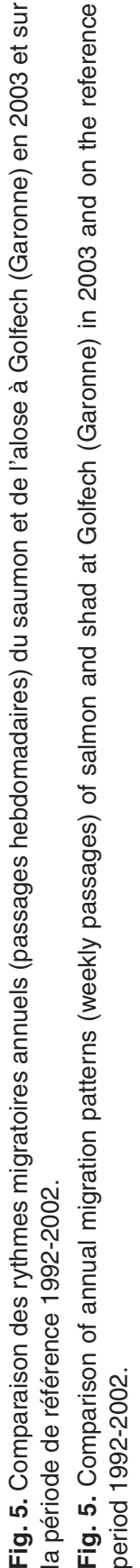



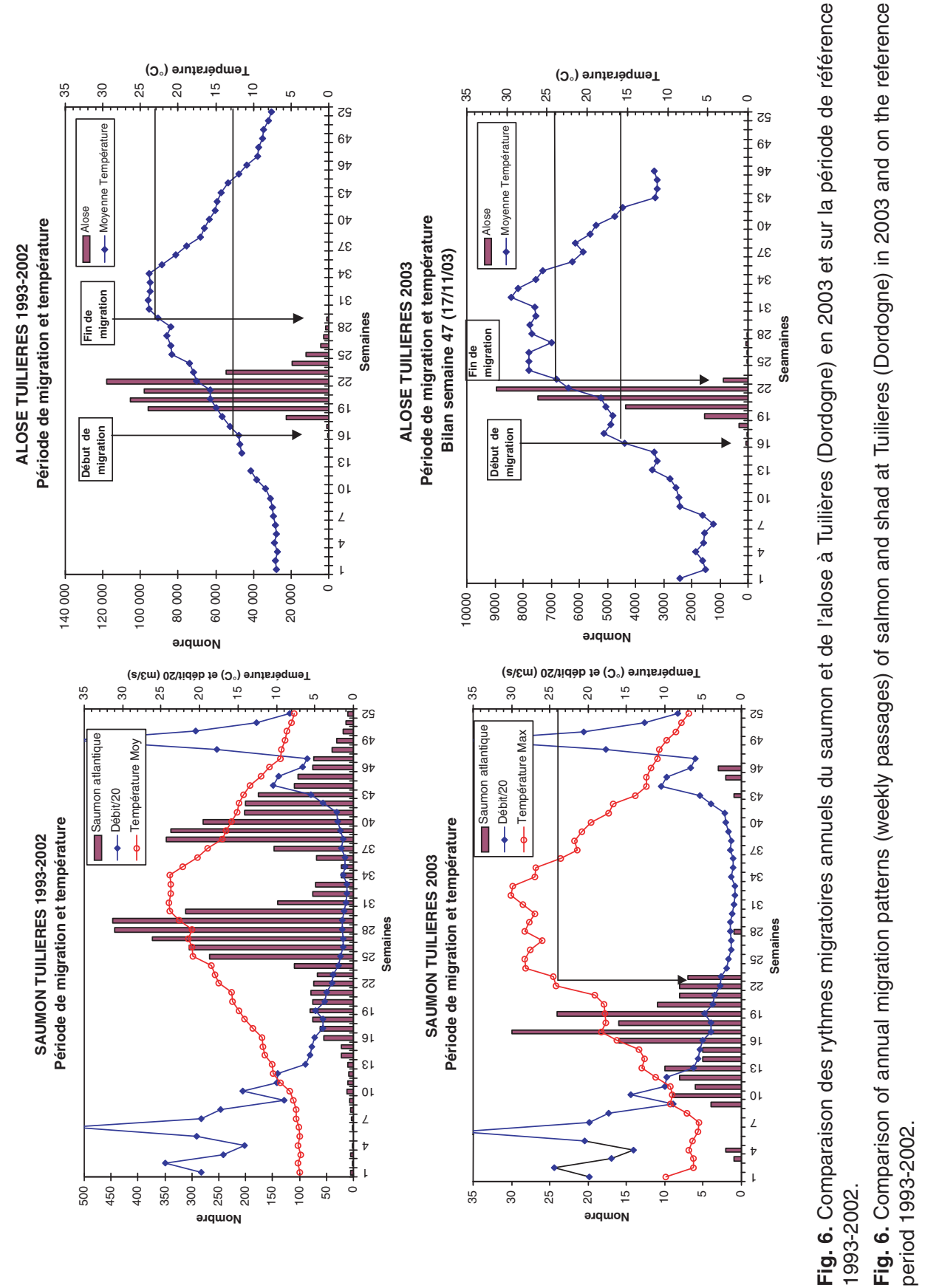
régimes hydrologiques et thermiques propres à chacun des deux cours d'eau.

En 2003, les phénomènes sont similaires sur les deux sites et caractérisés par une absence totale de migration d'été et une quasi absence de migration automnale.

À Golfech, la migration d'hiverprintemps (grands saumons) s'arrête la semaine 25 (18-24 juin) (Fig. 5), le dernier saumon passe le 18 juin avec une température de Garonne comprise entre $23^{\circ} \mathrm{C}$ et $24^{\circ} \mathrm{C}$ dans les jours précédant l'arrêt migratoire. À Tuilières la migration se termine le 9 juin (semaine 23) (Fig. 6) avec des températures similaires à celles de Golfech (23 à $24^{\circ} \mathrm{C}$ depuis le $1^{\mathrm{er}}$ juin).

La migration d'automne est quasi inexistante sur la Garonne (passage d'un seul saumon le 12 décembre) et très faible sur la Dordogne où seuls 6 individus ont été comptabilisés entre le 22 octobre et le 17 novembre.

II y a de fortes suspicions de mortalité d'au moins une partie de la population en rivière comme en témoignent d'une part les observations visuelles de saumons au pied du barrage de Tuilières début-juin qui ne sont plus vus ensuite ni au cours de l'été, ni à l'automne et d'autre part la mortalité de plus de $90 \%$ des saumons lors de l'opération de radiopistage conduite au printemps et en été 2003 sur la Garonne en amont de Golfech (Croze, 2003).

\subsection{Alose}

À l'échelle annuelle (Tabs. I et II, Figs. 4a et 4b), les passages d'aloses sur la période de référence
(1989-2002) sont similaires pour la Garonne et la Dordogne et se caractérisent par une augmentation graduelle des migrations jusqu'en 1996 (respectivement 106500 et 87300 individus à Golfech et Tuilières) puis une décroissance pour atteindre respectivement 17500 et 39500 individus sur les deux sites en 2002. En 2003, la migration sur les deux sites reste du même ordre de grandeur que celle de l'année 2002 : respectivement 22300 et 23800 individus sur les deux sites, soit, par rapport à l'année 2002, une légère augmentation des effectifs à Golfech (facteur 1,3 ) et une légère diminution à Tuilières (facteur 1,7 ).

À l'échelle hebdomadaire, on observe, sur la période de référence (Figs. 5 et 6), que la migration de l'alose sur les deux cours d'eau, se déroule en une seule période printanière comprise entre mi-avril (semaine 16) et mi-juillet (semaine 28), soit sur une durée de 12 semaines environ. Sur les deux sites, la fin de la période de migration semble correspondre à des températures voisines de 24 à $25^{\circ} \mathrm{C}$.

En 2003, les phénomènes sont similaires sur les deux sites (Figs. 5 et 6), et caractérisés par une période de migration très courte (semaines 17 à 25 , soit 8 semaines à Golfech et semaine 18 à 23 soit 5 semaines à Tuilières) stoppée dans le courant du mois de juin, dès que les températures de l'eau atteignent 23 à $24^{\circ} \mathrm{C}$.

Outre l'arrêt de migration, il semblerait qu'il y ait eu, sur la Garonne, des mortalités de géniteurs avant la reproduction comme en témoignent des autopsies pratiquées par MIGADO (L. Carry, comm. pers.), et l'ENSAT (2003) mais le phénomène n'a pas été quantifié. Aucune autopsie n'a été pratiquée sur la Dordogne mais l'on 
observait dès la mi-juin des mortalités de géniteurs plus importantes qu'à l'habitude au niveau de Bergerac, ce qui laisse suspecter un phénomène identique à celui de la Garonne.

Par ailleurs, la dévalaison des alosons, à l'automne 2003, semble avoir été très faible comme en témoigne le suivi estuarien réalisé, en Gironde, par le Cemagref (Girardin et al., 2004).

\subsection{Anguille}

À l'échelle annuelle (Tabs. I et II, Figs. $4 a$ et $4 b)$, les passages d'anguilles sur la période de référence ne sont pas interprétables dans la mesure où la station de comptage de Golfech n'était pas opérationnelle pour cette espèce jusqu'en 2002 et dans la mesure où, sur les deux sites, de nouvelles passes spécifiques à l'espèce (passes de reptation), beaucoup plus efficaces que les ascenseurs à poissons, ont été mises en service en 1997 à Tuilières et 2002 à Golfech. En 2003, on note toutefois que les passages d'anguille sont, par rapport à 2002, en nette augmentation à Golfech (102 000 individus en 2003 et 31000 en 2002) et similaires à Tuilières (environ 33000 individus en 2003 et 2002).

À l'échelle hebdomadaire, sur la période de référence (Figs. 7 et 8), la migration de l'anguille se déroule de façon similaire sur les deux sites avec une seule période de migration au printemps-été comprise entre mi-mai (semaine 19) et fin-août (semaine 35), soit sur une durée de 16 semaines. En 2003, le déroulement de la migration est similaire sur les deux sites et semblable à celui observé sur l'alose, c'est-à-dire caractérisé par une réduction très importante de la durée de migration avec des passages significatifs sur 7 semaines à Golfech (semaines 22 à 28, soit de débutjuin au 15 juillet) et sur 5 semaines à Tuilières (semaines 22 à 27 , soit de début-juin à début-juillet). L'arrêt de migration correspond à des températures de l'ordre de $26{ }^{\circ} \mathrm{C}$ sur les deux sites.

II n'existe aucune donnée ou observation de terrain permettant de savoir s'il y a eu ou non des mortalités d'anguille dans les deux cours d'eau. En tout cas, aucune observation visuelle de mortalité d'anguilles jaunes ou argentées n'a été rapportée sur les deux bassins comme cela avait été le cas sur le bassin de la Loire (COGEPOMI Loire, 2003) ou du Rhin (S. Manné, comm. pers.)

\subsection{Lamproie marine}

À l'échelle annuelle (Tabs. I et II, Figs. $4 a$ et $4 b$ ), les passages de lamproie sur la période de référence sont caractérisés par une forte variabilité interannuelle avec une abondance maximale de 4000 individus à Golfech et 11000 individus à Tuilières. En 2003, on constate une augmentation très nette des passages sur les deux sites (18 000 et 30000 individus respectivement à Golfech et Tuilières) qui représentent des records d'abondance sur la série chronologique. Dans les années suivant la canicule, on note une progression des abondances sur la Dordogne et une régression sur la Garonne.

À l'échelle hebdomadaire, sur la période de référence (Figs. 7 et 8), la lamproie marine migre en une seule période printanière d'une durée de 10 à 12 semaines comprise entre fin-avril 

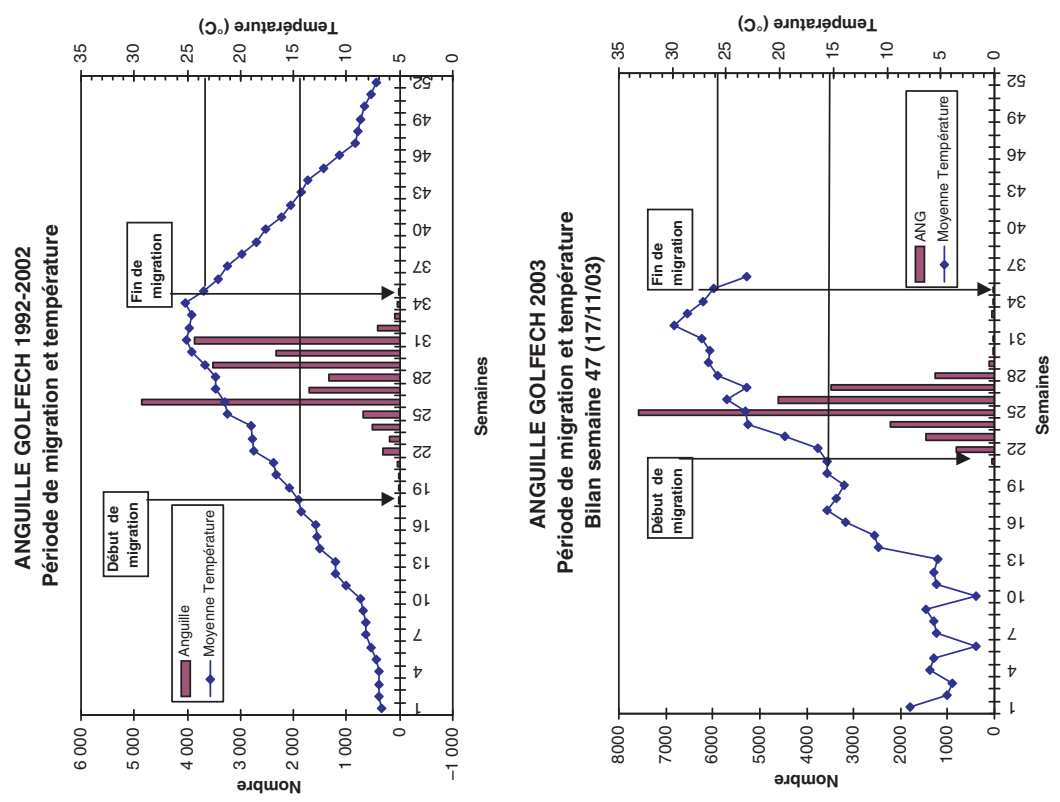

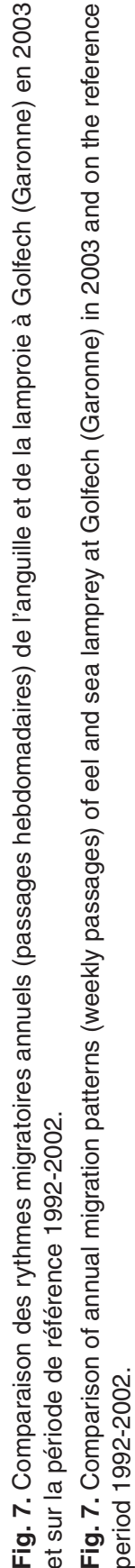
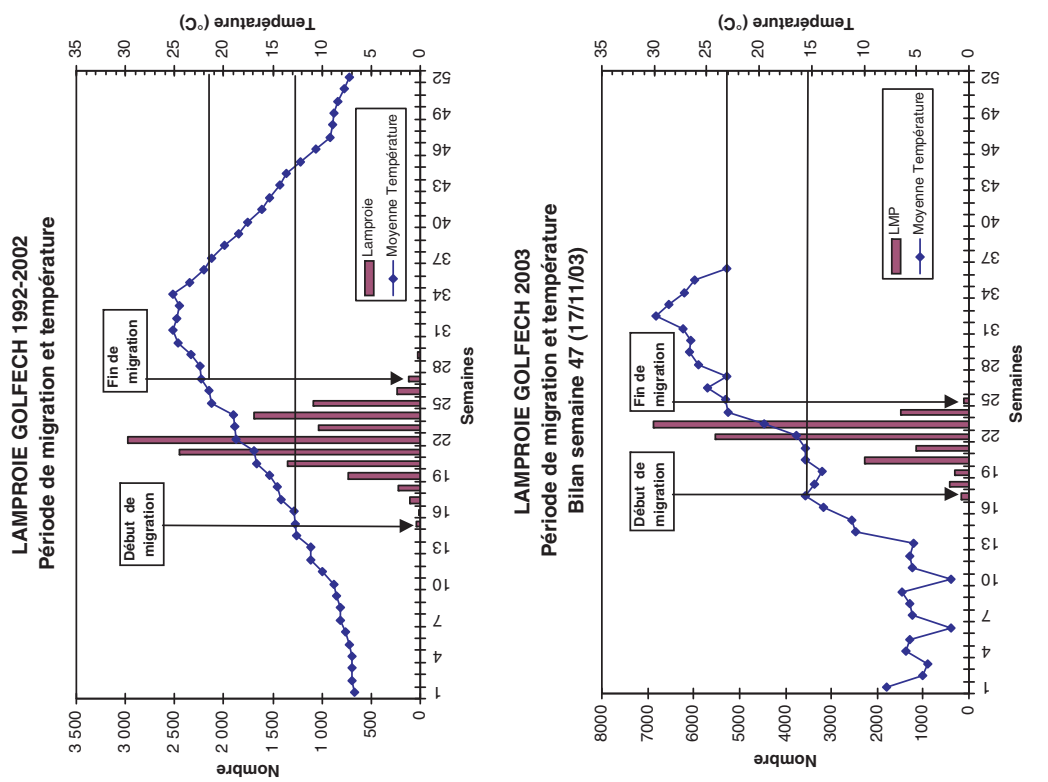

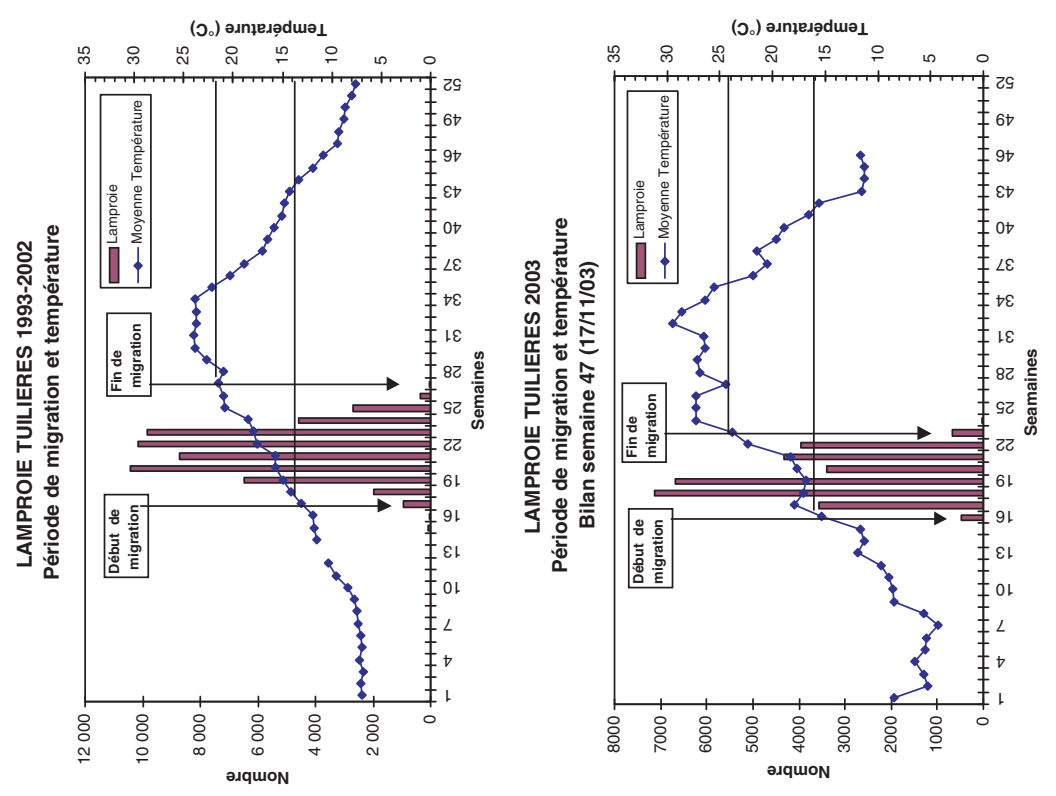

政
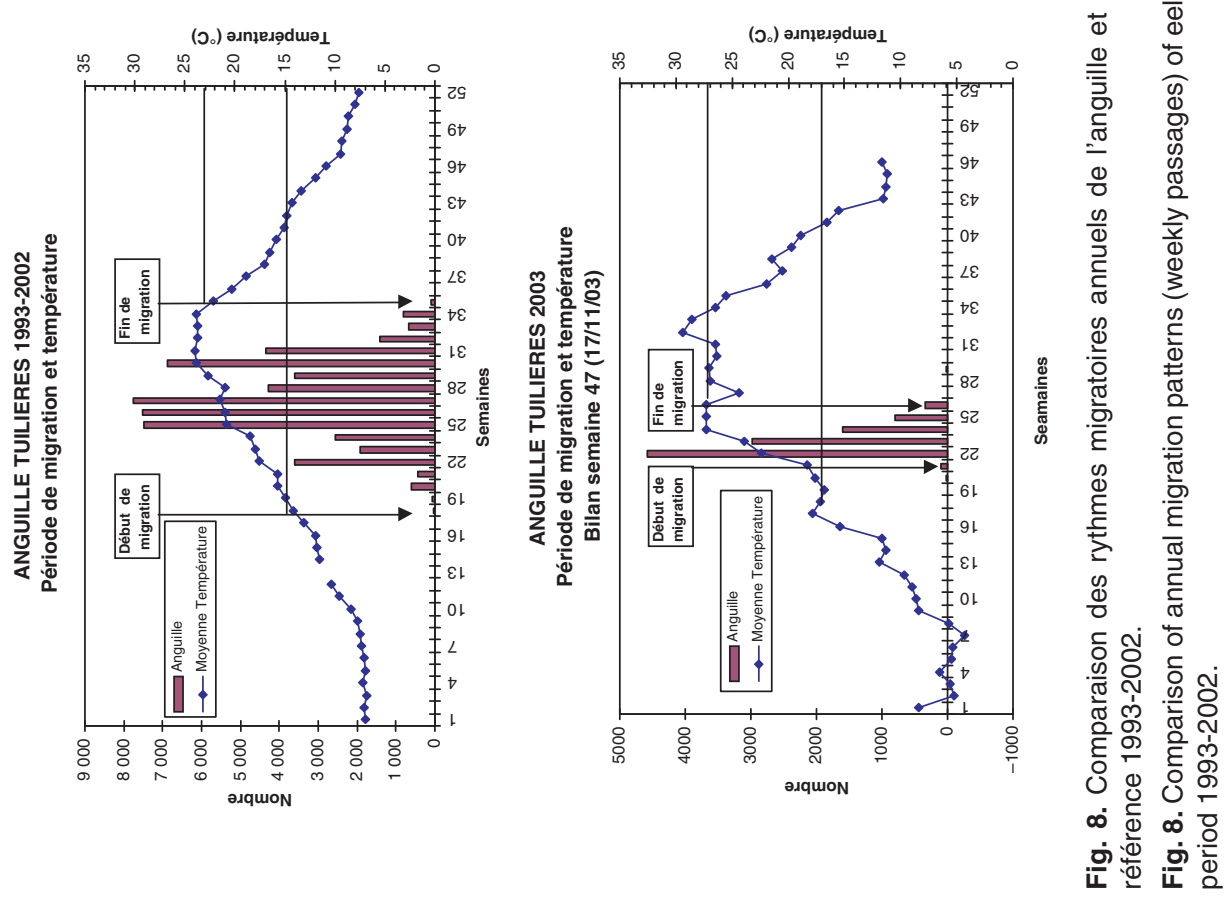
(semaine 17) et fin-juin (semaine 26). Cette période est similaire sur les deux sites. En 2003, on observe une migration écourtée d'environ 2 à 3 semaines sur les deux sites, avec une fin de migration semaine 24 (mi-juin) à Golfech et semaine 23 (10 juin) à Tuilières. Ces dates correspondent à des températures maximales de l'ordre de $24^{\circ} \mathrm{C}$.

Aucune information sur d'éventuelles mortalités dans les deux cours d'eau ni sur le succès de la reproduction n'est disponible. Comme à l'habitude, il a été observé de nombreux géniteurs morts sur les berges mais il s'agit d'un phénomène normal puisque la quasi-totalité des géniteurs de cette espèce meurt après la reproduction. Aucune autopsie de géniteurs n'a été réalisée sur ces cadavres pour voir s'il y avait eu, comme pour l'alose, des mortalités avant la reproduction.

\section{DISCUSSION - CONCLUSION}

Les données de passages dans les stations de contrôle de migration et les observations relatives aux quatre espèces de migrateurs diadromes, saumon Atlantique, alose, anguille et lamproie sur la Garonne et de la Dordogne lors de la canicule de 2003 permettent de mettre en évidence ou de suspecter plusieurs phénomènes : modifications des rythmes saisonniers de migration, évolution de la population migrante par rapport aux années antérieures et mortalités en rivière.

\subsection{Rythmes saisonniers}

Des modifications des rythmes saisonniers de migration au cours de l'année 2003 sont observées sur les quatre espèces et se traduisent essentiellement par un arrêt précoce des migrations, en avance de 2 à 4 semaines par rapport à la normale selon les espèces. Ce blocage migratoire est identique sur la Garonne et la Dordogne. Bien qu'il soit difficile de savoir si cet arrêt précoce est dû à un effet du débit fluvial, de la température de l'eau ou d'une synergie des deux paramètres, il est vraisemblable que la température de l'eau a joué un rôle prépondérant. En effet, sur les deux axes fluviaux, les arrêts migratoires se sont produits dès que les températures dépassent les mêmes seuils de 23 à $24^{\circ} \mathrm{C}$ pour le saumon, l'alose et la lamproie et de $26^{\circ} \mathrm{C}$ pour l'anguille. Ces températures d'arrêts de migration sont voisines de celles mises en évidence par Chanseau et al. (2000) sur l'alose, la lamproie et l'anguille lors de l'analyse des passages de migrateurs dans les stations de contrôle de Tuilières (1989-1999) et Golfech (1987-1999) : seuil d'arrêt de migration de l'alose de $23-24^{\circ} \mathrm{C}$ et $25^{\circ} \mathrm{C}$ respectivement à Tuilières et Golfech, de 22 et $23^{\circ} \mathrm{C}$ respectivement sur les deux sites pour la lamproie et de $26^{\circ} \mathrm{C}$ pour l'anguille. Pour le saumon, il a été observé par ces auteurs un seuil thermique légèrement plus élevé que celui observé en 2003, avec une température limite de migration de $25-26^{\circ} \mathrm{C}$ et des passages isolés dans les stations de contrôle à $26-27^{\circ} \mathrm{C}$. Ce seuil d'arrêt de migration est similaire à celui relevé sur l'Allier à Vichy (1998-2004) où la température maximale de passage dans la passe à poisson est de $25,9^{\circ} \mathrm{C}$ (Bach et al., 2005). Pour le saumon en Garonne et Dordogne on remarque par ailleurs qu'en 2003 la migration annuelle est quasiment stoppée dès le début de l'été et ne reprend pas à l'automne comme à l'habitude. Cela 
pourrait s'expliquer par des mortalités estivales ou des retours en mer des saumons entrés en rivière avant l'arrêt estival de migration.

\subsection{Population migrante annuelle}

L'impact de la canicule sur la population migrante annuelle n'est visible que sur le saumon où une réduction très nette des effectifs par rapport à l'année 2002 est observée sur les deux cours d'eau : facteur 4 sur la Garonne et 8 sur la Dordogne. Pour les trois autres espèces (alose, anguille, lamproie), les effectifs sont soit stables soit en augmentation par rapport à l'année 2002.

Pour le saumon, il est vraisemblable que ces déficits migratoires résultent en partie d'un blocage des migrations dans l'estuaire par les températures élevées et en partie de mortalités en rivière, à l'aval des stations de comptages. II est impossible de savoir lequel de ces facteurs est prépondérant, mais il est certain que les populations migrantes des deux axes sont particulièrement sensibles au facteur thermiques du fait que la population de saumon ayant passé 1 hiver en mer (1 HM ou castillons), dont la migration est estivale, y est majoritaire (75\%) (Chanseau et al., 2000). Les populations de saumon sur les axes fluviaux tels que la Loire où la majorité des individus ont 2 ou 3 ans de mer, caractérisées par une migration hivernale et printanière, n'ont pas été affectées de la même manière par la canicule que sur la Garonne et la Dordogne. Sur la Loire on note même une augmentation très nette des passages à la passe à poissons de Vichy (Allier) en 2003
(1238 individus) par rapport aux années précédentes (maximum de 541 individus en 2002 sur la période 19962002). L'ensemble de la population, composée à plus de $98 \%$ de saumons de 2 ou 3 hivers de mer est cependant passée à Vichy, comme tous les ans de janvier à juin, avant la période de canicule (Bach et al., 2004, 2005).

\subsection{Mortalités}

Le fait qu'aucune mortalité massive sur les quatre espèces diadromes considérées n'ait été observée ne signifie pas qu'il y ait eu absence de mortalité durant l'épisode de canicule. Les hypothèses de mortalités sont à discuter espèce par espèce à la lumière des informations ponctuelles recueillies durant l'épisode.

Pour le saumon, il y a de fortes suspicions de mortalité d'au moins une partie de la population des géniteurs en rivière. En effet, sur la Dordogne, plusieurs saumons ont été observés (comme à l'habitude) débutjuin au pied du barrage de Tuilières après l'arrêt estival des migrations dans l'ascenseur et ces individus ne sont passés dans l'ascenseur ni au cours de l'été, ni au cours de l'automne. Cette disparition pourrait s'expliquer soit par la mort des individus, soit par une dévalaison vers la mer, ce qui est peu probable pour des individus ayant séjourné plusieurs semaines en eau douce. Le fait que la migration automnale ait été absente (ou quasi-absente) sur les deux axes conforte cette hypothèse de mortalité estivale des individus entrés en eau douce au printemps et qui habituellement stationnent à l'aval de 
Golfech et Tuilières durant l'été avant de reprendre leur migration à l'automne. La deuxième information qui étaye cette hypothèse, est la mortalité de plus de $90 \%$ des saumons lors de l'opération de télémétrie (radiopistage) conduite par le GHAAPPE au printemps et en été sur la Garonne en amont de Golfech (Croze, 2003). Cette mortalité est intervenue alors que les températures de la Garonne excédaient $24^{\circ} \mathrm{C}$ (Croze et al., 2006, 2007). Cette opération de télémétrie, comme celles conduites auparavant sur d'autres axes (Gave de Pau, Loire...) a montré par ailleurs que les saumons morts restent en général immergés dans des zones où il est impossible de les retrouver de façon fortuite (O. Croze et J.M. Bach, comm. pers.), ce qui rend les mortalités difficilement détectables par de simples observations visuelles.

Pour l'alose, les mortalités d'adultes après la reproduction, mises en évidence par des échouages sur les berges ou par des poissons flottant à la surface de l'eau, sont habituelles tant sur la Garonne que sur la Dordogne du fait que la plupart des géniteurs ne survivent pas après la reproduction. En 2003, des mortalités d'adultes avant la reproduction ont été mises en évidence, fin-juin, sur la Garonne à l'aval de Golfech par autopsie de quelques individus échoués qui n'avaient pas encore pondu. Pour l'ENSAT (2003) et Carry (2004), ces mortalités ne résulteraient pas de températures excessives mais du gradient thermique exceptionnel de la première quinzaine de juin où la température est passée, en quelques jours, de $15^{\circ} \mathrm{C}$ à $25^{\circ} \mathrm{C}$, température au-delà de laquelle la reproduction de l'alose est stoppée (Cassou-Leins et Cassou-Leins,
1981). Ces autopsies, réalisées sur quelques individus seulement, ne permettent pas de quantifier cette mortalité pré-reproduction sur la Garonne ni de déterminer si de telles mortalités se sont produites sur la Dordogne où aucune autopsie n'a été pratiquée. L'observation, dès la mi-juin, au niveau de Bergerac de mortalités de géniteurs plus importantes qu'à l'habitude laisse cependant à penser que ce phénomène aurait pu avoir lieu également sur la Dordogne. Les éventuelles mortalités des œufs, larves et juvéniles d'alose suite aux fortes températures de juillet et août (égales ou supérieures à $30^{\circ} \mathrm{C}$ ) n'ont pas été inventoriées; par contre les pêches d'alosons pratiquées par l'ENSAT, en septembre et octobre 2003, dans les chambres d'équilibre de la centrale hydroélectrique de Golfech, ont montré que ces juvéniles avaient eu une croissance supérieure à celle observée dans les années 1995 à 1997. De manière globale, il semble cependant que la dévalaison des alosons ait été très faible en 2003 puisque les inventaires réalisés dans l'estuaire de la Gironde par le Cemagref à l'automne dans le cadre du programme de surveillance halieutique de l'estuaire (Girardin et al., 2004) font apparaître une abondance " proche de zéro jamais observée auparavant ». II est cependant impossible de savoir si la faiblesse de cette cohorte résulte d'un déficit de reproduction par mortalité des adultes avant reproduction ou de mortalités anormalement élevées des stades juvéniles.

Pour l'anguille, aucune mortalité anormale n'a été signalée sur les bassins de la Garonne et de la Dordogne alors que des mortalités ont été observées sur divers bassins 
et en particulier ceux de la Loire (COGEPOMI Loire, 2003) et du Rhin (S. Manné, comm. pers.). Sur ces bassins il s'agissait principalement d'individus de grande taille, en majorité des femelles en cours d'argenture. Les causes de mortalités avancées sont floues: parasitisme, accumulation de toxiques chimiques, stress thermique, bactériose sur la Loire, bactériose (Aeromonas) et virose (virus HVA) sur le Rhin.

Pour la lamproie, comme pour l'alose, les cadavres de géniteurs qui meurent naturellement après la reproduction sont observés couramment sur les berges des deux cours d'eau. En l'absence d'autopsie, il est impossible de savoir si, comme pour l'alose, une fraction des géniteurs est morte avant la reproduction. II apparaît en tout cas qu'une partie au moins de la reproduction de l'espèce ait été assurée normalement au vu des nombreux « nids » de ponte observés par l'ENSAT (2003) à l'aval de Golfech et en particulier à La Magistère, Saint-Sixte et entre Saint-Pierre de Gaubert et le pont de Leyrac. Aucune investigation quantitative n'a par ailleurs été, à notre connaissance, réalisée sur les larves ammocètes pour juger du succès de la reproduction.

La question de l'impact supplémentaire des rejets thermiques de la centrale nucléaire de Golfech sur les quatre espèces de migrateurs diadromes considérées dans le contexte de la canicule de 2003 n'est pas chose aisée car une grande partie des données reste parcellaire. La similarité de l'ensemble des phénomènes sur les deux sites dont l'un est influencé par des rejets thermiques de la centrale nucléaire (Golfech) et l'autre pas (Tuilières) laisse toutefois à penser que l'influence de la centrale de Golfech sur les migrations de ces espèces a été vraisemblablement mineure voire inexistante au cours de cet épisode particulier.

Au vu des phénomènes observés, et compte tenu des incertitudes quant à l'effet exact de la canicule sur les migrateurs et des perspectives d'augmentation de la fréquence d'épisodes caniculaires dans le contexte du changement climatique, une attention toute particulière devrait être accordée, à l'avenir, aux points suivants (liste non exhautive) :

- poursuivre le suivi régulier de l'évolution des diverses populations de migrateurs sur les principaux axes fluviaux en France de façon à mettre en évidence les éventuels effets différés de la canicule de 2003 ainsi que la réponse de ces populations au changement climatique,

- mettre en œuvre très rapidement, lors de tout nouvel épisode caniculaire, les investigations de terrain adaptées à la compréhension des effets des températures élevées sur les migrateurs à leurs divers stades de développement,

- réaliser des études expérimentales sur la sensibilité thermique de l'alose aux stades œufs, larves et juvéniles les plus exposés aux fortes températures estivales.

\section{RÉFÉRENCES BIBLIOGRAPHIQUES}

Bach J.M., Lelievre M., Postic A., Viallard J., Bomassi P. \& Brugel C., 2004. Sauvegarde du grand saumon de Loire. 
Programme LIFE00NAT/F/7252. Rapport d'avancement couvrant la période du $1^{\mathrm{er}}$ janvier 2003 au $1^{\mathrm{er}}$ mars 2004. $69 \mathrm{p}$.

Bach J.M., Lelievre M., Postic A., Viallard J., Bomassi P. \& Brugel C., 2005. Sauvegarde du grand saumon de Loire. Programme LIFE00NAT/F/7252. Rapport Technique Final d'Activité 2001-2004. 81 p.

Baglinière J.L. \& Porcher J.P., 1994. Caractéristiques des stocks de reproducteurs et comportement lors de la migration génésique. Le saumon Atlantique, J.C. Gueguen \& P. Prouzet (Eds.). Editions de l'IFREMER, centre de Brest. ISBN 2-905434-48-1, pp. 101-122.

Carry L., 2004. Suivi de la reproduction de la grande alose sur la moyenne Garonne et l'axe Tarn-Aveyron en 2003. Rapport MIGADO. 30 p.

Cassou-Leins F. \& Cassou-Leins J.J., 1981. Recherches sur la biologie et I'halieutique des migrateurs de la Garonne et principalement de l'Alose, Alosa alosa L. Thèse doct. Instit. Nat. Polytech. Toulouse. 382 p. + annexes.

Chanseau M., Dartiguelongue J. \& Larinier M., 2000. Analyse des données sur les passages enregistrées aux stations de contrôle des poissons migrateurs de Golfech et du Bazacle sur la Garonne et de Tuilières sur la Dordogne. Rapport GHAAPPE RA00.12 / MIGADO G14-00-RT, $64 \mathrm{p}$.

COGEPOMI Loire, 2003. Bilan de l'étiage 2003 sur le territoire du COGEPOMI du bassin de la Loire et des côtiers vendéens. Note COGEPOMI. 19 p.

Croze O., 2003. Note relative à l'impact de la canicule de l'été 2003 sur la population de saumons radiomarqués en Garonne. Note GHAAPPE septembre 2003. 4 p.

Croze O., Blot E., Delmas F., Jourdan H., Bau F. \& Breinig T., 2006. Suivi de la qualité de l'eau de Garonne lors de la migration anadrome du saumon en amont de Golfech. Rapport GHAAPPE RA06.04, $156 \mathrm{p}$.

Croze O., Blot E. \& Delmouly L., 2007. Évolution de la température de l'eau de la Garonne au cours des 3 dernières décennies (1977-2005). Rapport GHAAPPE RA07.04, 85 p.

ENSAT, 2003. Effet de la sécheresse de 2003 sur les poissons de la Garonne au niveau de Golfech. Note novembre 2003. 52 p.

Girardin M., Castelnaud G. \& Beaulaton L., 2004. Surveillance halieutique de l'estuaire de la Gironde - Suivi des captures 2002 - Étude de la faune circulante 2003. Rapport pour EDF CNPE du Blayais / Étude Cemagref, Groupement de Bordeaux Cestas $\mathrm{N}^{\circ} 91$. $201 \mathrm{p}$.

Hendrickx F., Khalanski M., Gosse Ph. \& Drouadaine L., 2003. Régime thermique de la Garonne à l'aval du CNPE de Golfech lors de la canicule d'août 2003. Rapport EDF R\&D HP76/03/046/A. 46 p.

MIGADOa, 1992 à 2007. Étude des rythmes de migration des espèces amphibiotiques et holobiotiques de la Garonne au niveau de la station de contrôle de Golfech. Rapports annuels MIGADO 1992 à 2007.

MIGADOb, 1992 à 2005. Étude des rythmes de migration des espèces amphibiotiques et holobiotiques de la Dordogne au niveau de la station de contrôle de Tuilières. Rapports annuels MIGADO 1992 à 2005. 
